\title{
Structure can predict function in the human brain: a graph neural network deep learning model of functional connectivity and centrality based on structural connectivity
}

\author{
Josh Neudorf ${ }^{1} \cdot$ Shaylyn Kress ${ }^{1} \cdot$ Ron Borowsky $^{1}$ (i) \\ Received: 13 May 2021 / Accepted: 30 September 2021 / Published online: 11 October 2021 \\ (c) The Author(s) 2021
}

\begin{abstract}
Although functional connectivity and associated graph theory measures (e.g., centrality; how centrally important to the network a region is) are widely used in brain research, the full extent to which these functional measures are related to the underlying structural connectivity is not yet fully understood. Graph neural network deep learning methods have not yet been applied for this purpose, and offer an ideal model architecture for working with connectivity data given their ability to capture and maintain inherent network structure. Here, we applied this model to predict functional connectivity from structural connectivity in a sample of 998 participants from the Human Connectome Project. Our results showed that the graph neural network accounted for $89 \%$ of the variance in mean functional connectivity, $56 \%$ of the variance in individuallevel functional connectivity, $99 \%$ of the variance in mean functional centrality, and $81 \%$ of the variance in individual-level functional centrality. These results represent an important finding that functional centrality can be robustly predicted from structural connectivity. Regions of particular importance to the model's performance as determined through lesioning are discussed, whereby regions with higher centrality have a higher impact on model performance. Future research on models of patient, demographic, or behavioural data can also benefit from this graph neural network method as it is ideally-suited for depicting connectivity and centrality in brain networks. These results have set a new benchmark for prediction of functional connectivity from structural connectivity, and models like this may ultimately lead to a way to predict functional connectivity in individuals who are unable to do fMRI tasks (e.g., non-responsive patients).
\end{abstract}

Keywords Functional connectivity · Resting state fMRI $\cdot$ Structural connectivity $\cdot$ DTI $~$ Deep learning · Centrality

\section{Introduction}

There is now widespread usage of functional connectivity and associated graph theory measures (e.g., centrality). Investigating to what extent the structural connectivity as measured by diffusion tensor imaging (DTI) can explain functional connectivity as measured by resting state functional magnetic resonance imaging (rsfMRI) is an important step towards understanding the structural basis of functional macroscale networks (the importance of this problem has recently been highlighted; Suárez et al. 2020). Some of the first steps towards understanding the relationship between

Ron Borowsky

ron.borowsky@usask.ca

1 Cognitive Neuroscience Lab, Department of Psychology, University of Saskatchewan, Saskatoon, SK, Canada structure and function showed moderate correspondence when examining direct structural connections, accounting for approximately 50\% of the variance (Honey et al. 2009), or looking at a subset of connections (62\%; Hagmann et al. 2008). However, when examining all functional connections, structural connectivity accounts for only $9 \%$ of the variance using linear regression by one account (Rosenthal et al. 2018). Novel graph theory measures calculated using structural connectivity have accounted for $23 \%$ of the variance in functional connectivity (Goñi et al. 2014), a combination of vector encodings of structural connectivity and deep learning with a fully connected network (FCN) have accounted for $36 \%$ of the variance in functional connectivity (Rosenthal et al. 2018), and simulated fMRI activation using a hybrid approach with both DTI and electroencephalography (EEG) data has accounted for $53 \%$ of the variance in functional connectivity (Schirner et al. 2018). Still, there remains a large amount of variance unaccounted for by these models if we 
are to determine the extent to which functional connectivity and measures of centrality based on functional connectivity offer insight into the properties of the underlying structural network. Recently, a feed-forward FCN deep learning model was able to demonstrate that mean structural connectivity as input could predict mean functional connectivity accounting for $81 \%$ of the variance, and that individual-level structural connectivity could predict individual-level functional connectivity accounting for $30 \%$ of the variance (Sarwar et al. 2021). These findings represent a large improvement in prediction of functional connectivity. Further deep learning models should be investigated to determine whether there is a converging upper limit on functional connectivity prediction, in order to provide a benchmark to work towards with more explicit mathematical and simulation methods.

There is a general consensus in the neuroscience community that rsfMRI functional connectivity measures represent the effective connectivity between regions in the brain. Effective connectivity describes the meaningful result of communication between regions that are sparsely connected by direct and indirect structural connections (white matter tracts). To support this view researchers have noted that patterns of connectivity seem to occur between regions that are expected to function together based on previous research and neuroanatomy (see van den Heuvel and Hulshoff Pol 2010 for a review). Modern preprocessing methods are able to account for the effects of physiological factors (e.g., respiration and cardiac function), in order to increase the neuronal basis of the BOLD signal relative to noise (e.g., Birn et al. 2008; Chang et al. 2009; Falahpour et al. 2013; Golestani et al. 2015; Kassinopoulos and Mitsis 2019; Salas et al. 2021). Even with these advances, coordinated functional BOLD signal (functional connectivity) has yet to be robustly linked to the organization of the underlying structural network. Searching for the upper limit in predicting functional connectivity from structure remains an important goal for investigating to what extent the rsfMRI functional connectivity is influenced by the connectivity of the structural architecture of the brain.

One issue with structural connectivity is that DTI tractography data is sparse, meaning the majority of values are zeros. On the other hand, functional connectivity data has many more non-zero values, even when thresholded. This difference occurs in part because many routes of communication between brain regions are indirect rather than direct, and is one reason functional connectivity has been widely used. Measures of indirect ('effective') connectivity are available using graph theory, including shortest path length, communicability (Estrada and Hatano 2008), and novel measures designed for brain research (e.g., search information and path transitivity; Goñi et al. 2014), but research is still investigating the extent to which these measures are based on sound assumptions about how functional connectivity results from underlying structure at the macroscale.

Research employing graph theory measures have become an important focus of recent network neuroscience research involving structural and functional connectivity (see Fornito et al. 2013), including measures of centrality, which describe a region's importance to the network. Some common centrality measures include degree centrality (number of connections to a region), eigenvector centrality (number of connections to a region weighted by the centrality of its neighbours), and PageRank centrality (a variant of eigenvector centrality developed for use in ranking web pages, with the advantage that it addresses the issue of eigenvector centrality sometimes being excessively high when a low degree node is connected to a high centrality node; Page et al. 1999). Figure 1A depicts an example graph with labels showing which node has the highest centrality for each of these measures. This example graph is relatively sparse (fewer connections) like the structural connectivity network of the brain, in comparison to functional connectivity networks, which are denser (highly connected). Functional connectivity centrality has been used to demonstrate age and sex related differences (Zuo et al. 2012), differences between patient and control groups (for patients with schizophrenia, Chen et al. 2015; bipolar disorder, Deng et al. 2019, Zhou et al. 2017; retinitus pigmentosa, Lin et al. 2021; and diabetic optic neuropathy, $\mathrm{Xu}$ et al. 2020), and differences related to genotype (Wink et al. 2018). Structural connectivity centrality has also been used to demonstrate differences between patient and control groups (for patients with prenatal alcohol exposure, Long et al. 2020; traumatic brain injury, Raizman et al. 2020; gut inflamation, Turkiewicz et al. 2021; and brain tumours, Yu et al. 2016), and to demonstrate a relationship between structural centrality and functional complexity (e.g., Hurst exponent; inversely related to fractal dimension, where a fractal dimension exceeding the topological dimension of the signal indicates complex functional activity) suggesting that regions integrating information from many sources have more complex functional activity (Neudorf et al. 2020). An important question that has not yet been explored, to our knowledge, is to what extent variance in functional connectivity-based centrality measures can be accounted for by structural connectivity and structural connectivitybased centrality.

The problem of developing a deep learning model approach to using brain connectivity for prediction has been a focus of recent research. In a graph neural network model for deep learning, the structure of the connectivity data as a network (graph) is maintained, making this model ideal for prediction problems related to connectivity data. One implementation of this model architecture (not using additional global values in this case), Graph Nets (Battaglia et al. 2018), trains a small FCN edge update function to update 


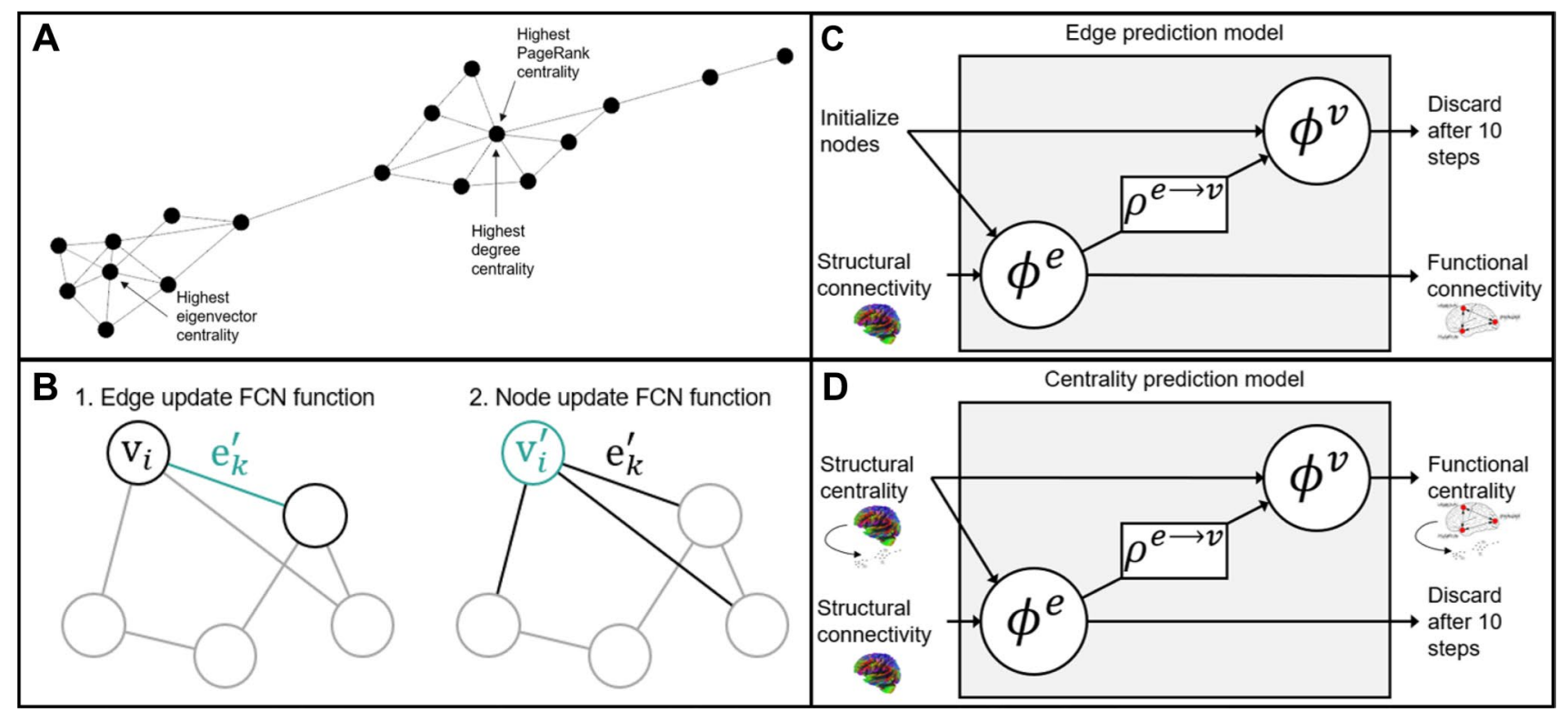

Fig. 1 Graph neural network deep learning architecture. A An example graph illustrating degree centrality, eigenvector centrality, and PageRank centrality. B Depiction of Graph Nets update functions, where $e_{k}^{\prime}$ refers to the updated edge value, and where $v_{i}$ and $v_{i}^{\prime}$ refer to the node being updated and then its updated value. Adapted from Battaglia et al. (2018). C and D Depiction of the steps in the edge

the value of each edge. This edge update function takes as input the current value of the edge as well as the values of each connected node. Another trainable FCN node update function updates the value of each node. This node update function takes as input the current value of the node as well as the aggregated value of all connected edges (see Fig. 1B). These two FCN functions can produce very good prediction results even at a very constrained scale, given only two layers with 16 nodes each (many fewer parameters to train than previous deep learning models predicting functional connectivity from structural connectivity; e.g., 4 layers with 350 nodes each in Rosenthal et al. 2018; 8 layers with 1024 nodes each in Sarwar et al. 2021), owing to the ability of the model to preserve the network structure of the data. Any number of updates can be performed, each using the updated values from the last step, before calculating the loss function and training the update functions (message passing). Graph neural network approaches to deep learning have been consistently outperforming other models of deep learning with brain connectivity data, as evidenced by higher prediction accuracy. Some applications of a graph neural network model to brain connectivity data include demonstrations of sex prediction from functional connectivity (88\% accuracy; Arslan et al. 2018), a similarity metric learning model for predicting the similarity between two functional connectivity networks (63\% accuracy; Ktena et al. 2017), prediction of Alzheimer's disease from functional connectivity that prediction model $(\mathbf{C})$, and centrality prediction model $(\mathbf{D})$, where $\phi^{e}$ represents the FCN update function for edges taking an edge and 2 connected nodes as input, $\phi^{v}$ represents the FCN update function for nodes taking a node and the aggregated value of connected edges as input, and $\rho^{e \rightarrow v}$ represents the aggregation of edge values. Adapted from Battaglia et al. (2018)

outperformed previous methods ( $81 \%$ accuracy; Bi et al. 2020; Parisot et al. 2018), and prediction of Autism Spectrum Disorder outperforming previous methods (61\% to 71\%; Arya et al. 2020; Parisot et al. 2018; Wang et al. 2021; Zhang and Wang 2020). To understand why graph neural network approaches have consistently outperformed other deep learning methods in neuroscience, it is important to understand that unlike FCN methods used previously, the graph neural network approach maintains the network structure of structural and functional connectivity. Conversely, the drawback of a FCN method is that all edge values are reduced to a single dimensional vector, which discards the vital network level patterns of connectivity. By maintaining the network structure, the graph neural network approach is able to produce superior performance using a much more constrained architecture with many fewer parameters (an edge and a node update function with only 2 layers of 16 nodes each, compared to FCN methods for example with 8 layers of 1024 nodes each). Additionally, because the graph neural network update functions apply the same function for every edge and node, this model applies a graph theory function that may represent a plausible explicit structure-function relationship to be explored with future research.

Considering the ultimate goal of relating DTI structural connectivity to resting state functional connectivity, a graph neural network deep learning approach has not yet been established, and based on past successes predicting other 
measures using brain connectivity this type of model is a promising candidate. This research uses a Graph Nets (Battaglia et al. 2018) deep learning model to determine to what extent structural connectivity can predict functional connectivity, as well as functional connectivity derived centrality measures.

\section{Methods}

\section{Dataset}

High quality DTI and resting state fMRI data for 998 subjects were obtained from the Human Connectome Project (HCP) database (Van Essen et al. 2013; please see this paper for ethics statements). The HCP preprocessed rsfMRI data was used, which has been FSL FIX (Salimi-Khorshidi et al. 2014) preprocessed, along with the DTI preprocessed data (see HCP preprocessing pipelines for more information on preprocessing steps, Glasser et al. 2013). The mean activation at each timepoint was calculated for each region of the Desikan-Killiany 66 region atlas (DK; Desikan et al. 2006; removed the corpus collosum region as in recent updates; e.g., Destrieux et al. 2010; Klein and Tourville 2012) and of the Automated Anatomical Labelling 90 region atlas (AAL; Tzourio-Mazoyer et al. 2002). The DK atlas was used in the previous best prediction attempt (Sarwar et al. 2021), and multiple atlases were examined as the structure-function relationship is known to be atlas dependent (Messé, 2020). Each of the 4 rsfMRI sessions was then z-score standardized for each region independently from each other session. Each region was then bandpass filtered for each session separately to keep frequencies between 0.01 and $0.1 \mathrm{~Hz}$ (see Hallquist et al. 2013).

\section{Connectivity measures}

The Pearson correlation coefficient was then calculated for each pair of regions using the 4800 total time points (all 4 sessions concatenated), as a measure of functional connectivity. DSI Studio's (http://dsi-studio.labsolver.org) deterministic tracking algorithm that uses quantitative anisotropy (Yeh et al. 2013) as the termination index was used to produce structural connectivity matrices of streamline count. For reconstruction in DSI Studio the Generalized Q-sampling (Yeh et al. 2010) method was used, and tracking was performed using a fiber count of 1 million fibers, maximum angular deviation of 75 degrees, and a minimum and maximum fiber length of $20 \mathrm{~mm}$ and $500 \mathrm{~mm}$ respectively. A whole brain seed was used to calculate the structural connectivity matrix as the count of streamlines between each combination of the atlas regions.
Graph theory centrality measures of degree centrality, eigenvector centrality, and PageRank centrality were calculated using the NetworkX python library (Hagberg et al. 2008; using functions degree, eigenvector_centrality, and pagerank). These measures were calculated using a thresholded functional connectivity matrix, with the lower threshold set to the critical correlation coefficient for $\mathrm{R}$ with $p=0.0001$ (see Zuo et al. 2012). Likewise, the DTI structural connectivity matrix was used to calculate degree, eigenvector, and PageRank centrality for each atlas region. The structural and functional centrality measures were then $z$ score standardized and rescaled to have values between -1 and 1 .

\section{Model architecture}

The Graph Nets (Battaglia et al. 2018) python library, which relies on Tensorflow (Abadi et al. 2015), was used with a message passing design, using 10 message passing steps (as in the default model defined by Battaglia et al. 2018; accessible at https://github.com/albertotono/graph_nets; the code used to apply this library is available upon reasonable request). Following Battaglia et al. (2018) two latent layers were used with 16 nodes in each layer for the update functions. Seventy percent of the data was used as a training set, with 10 percent used for validation during training and 20 percent saved for testing (as done previously; e.g., Wang et al. 2020). The Adam (Kingma and Ba 2017) optimizer was used with default values (learning rate $=0.001, \beta_{1}=0.9$, $\beta_{2}=0.999$, and $\varepsilon=1 * 10^{-7}$ ). Training was performed using batch gradient descent, with a batch size of 32 used for training the models. The loss function used was the mean squared error (MSE) of the functional measures compared to the predicted values. One epoch was completed when enough batches were completed to randomly sample as much unique test data as possible ( 21 batches for batch size of 32 ). For the edge prediction models, structural connectivity values were given as the input edges, all input nodes were initialized with a value of 1.0, and functional connectivity measures were compared to the output edges to calculate loss (see Fig. 1C). For the centrality prediction models, structural connectivity values were given as the input edges, structural centrality measures were given as the input nodes, and functional centrality measures were compared to the output nodes to calculate loss (see Fig. 1D). In order to encourage the model to find an effective solution in as few steps as possible, the loss was calculated as the mean MSE for all 10 message passing steps. A Nvidia GTX 3080 graphical processing unit (GPU) was used to train the models and required approximately $11 \mathrm{~h}$ to run 3000 epochs for the centrality prediction models and approximately $83 \mathrm{~h}$ to run 5000 epochs for the edge prediction model. 


\section{Results}

\section{Edges}

The edge prediction model was trained for 5000 epochs, and there was no evidence for overfitting of the model to the training set, as the validation set decreased its loss along with the training set (Figs. 2A, 3A). Figures 2B, 3B depict the empirical versus the mean predicted values of functional connectivity, accounting for $89.4 \%$ (DK) and $81.3 \%$ (AAL) of the variance in the 201 subject test group. Furthermore, functional connectivity was predicted at the individual-level accounting for $55.7 \%$ (DK) and $47.8 \%$ (AAL) of the variance (see Supplementary Figs. 1 and 2). The structural connectivity of each individual atlas region was iteratively lesioned, and this lesioned network was given to the previously trained model as input. The MSE lesioned loss was then calculated, whereby an increase in loss following a removal of that region indicates the importance of that region to model performance (see Figs. 2C, 3C). For the DK atlas the top 10 regions that impacted the model performance the most included bilateral superior frontal cortex, bilateral precentral gyrus, superior parietal lobe, insula, and lingual gyrus. For the AAL atlas the top 10 regions that impacted the model performance the most included the bilateral middle occipital gyrus, $\mathrm{RH}$ angular gyrus, $\mathrm{LH}$ precentral gyrus, $\mathrm{RH}$ superior frontal lobe, $\mathrm{RH}$ middle cingulum, RH olfactory cortex, $\mathrm{LH}$ insula, LH middle temporal pole, and RH lingual gyrus. In order to gain a better understanding of what features of the regions make them important for model performance, we tested a hypothesis that higher structural degree centrality (more centrally important to the network as a whole because of the high number of connections) may be related to higher impact on model performance. This was the case, as it was observed that the centrality of a region was positively related to lesion loss (Figs. 2D, 3D; DK: $R(64)=0.774, p<0.001$; AAL: $R(88)=0.322, p=0.002$ ), indicating that high centrality regions were more important for model performance (see Fig. 2D).

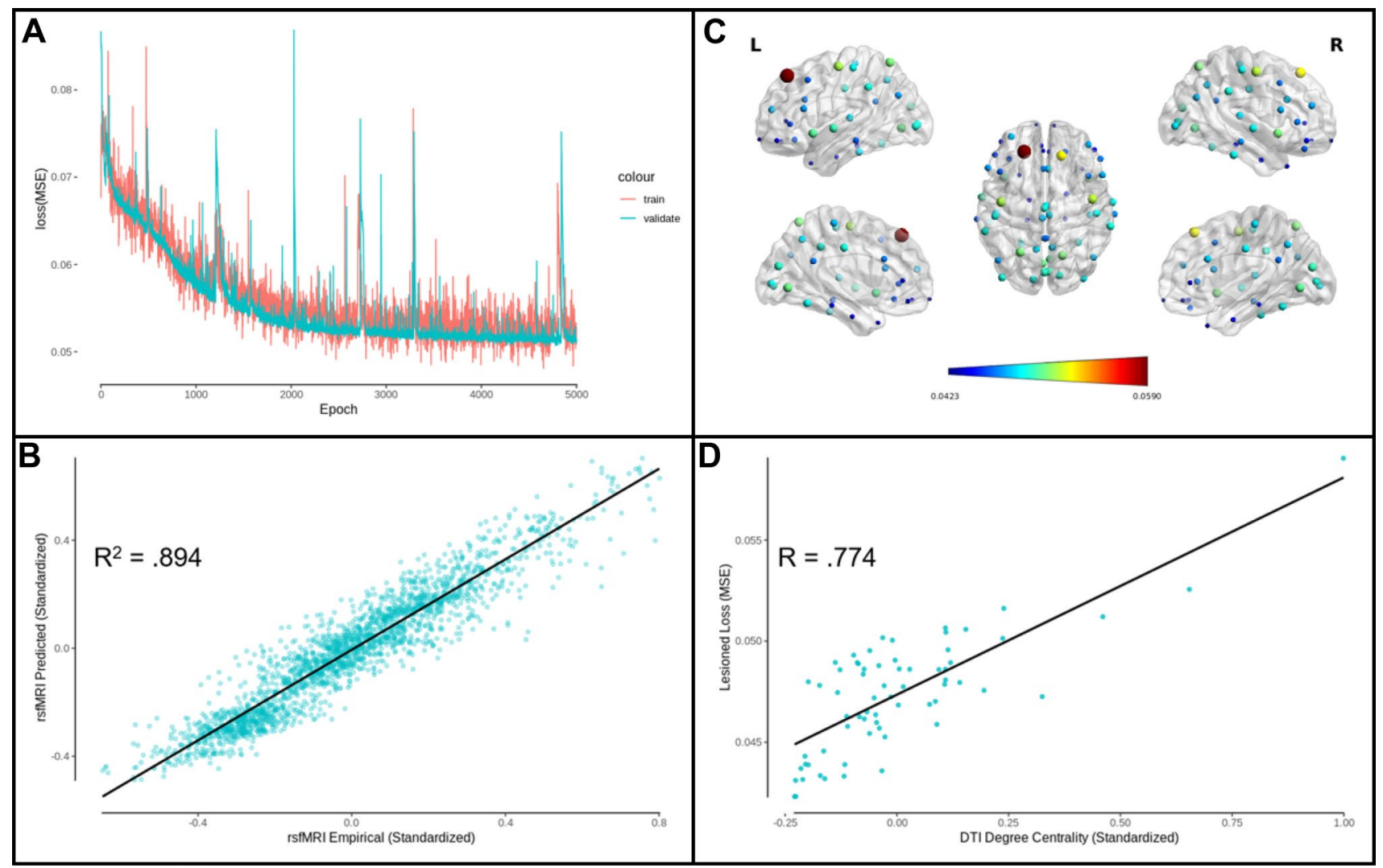

Fig. 2 DK atlas edge prediction model performance. A Edge prediction model MSE loss for training and validation sets as a function of the number of training epochs. B Predicted rsfMRI functional connectivity as a function of empirical rsfMRI functional connectivity $\left(R^{2}=0.894\right)$. C Functional connectivity loss (MSE) related to lesioning structural connectivity to each atlas region, where dark blue indi- cates a lesser effect on the model performance and dark red indicates a greater effect. Larger sphere size also indicates a greater effect of lesion on model performance. Figure produced using BrainNet Viewer (Xia et al. 2013). D Lesioned functional connectivity loss as a function of structural connectivity degree centrality $(R(64)=0.774$, $p<.001)$ 


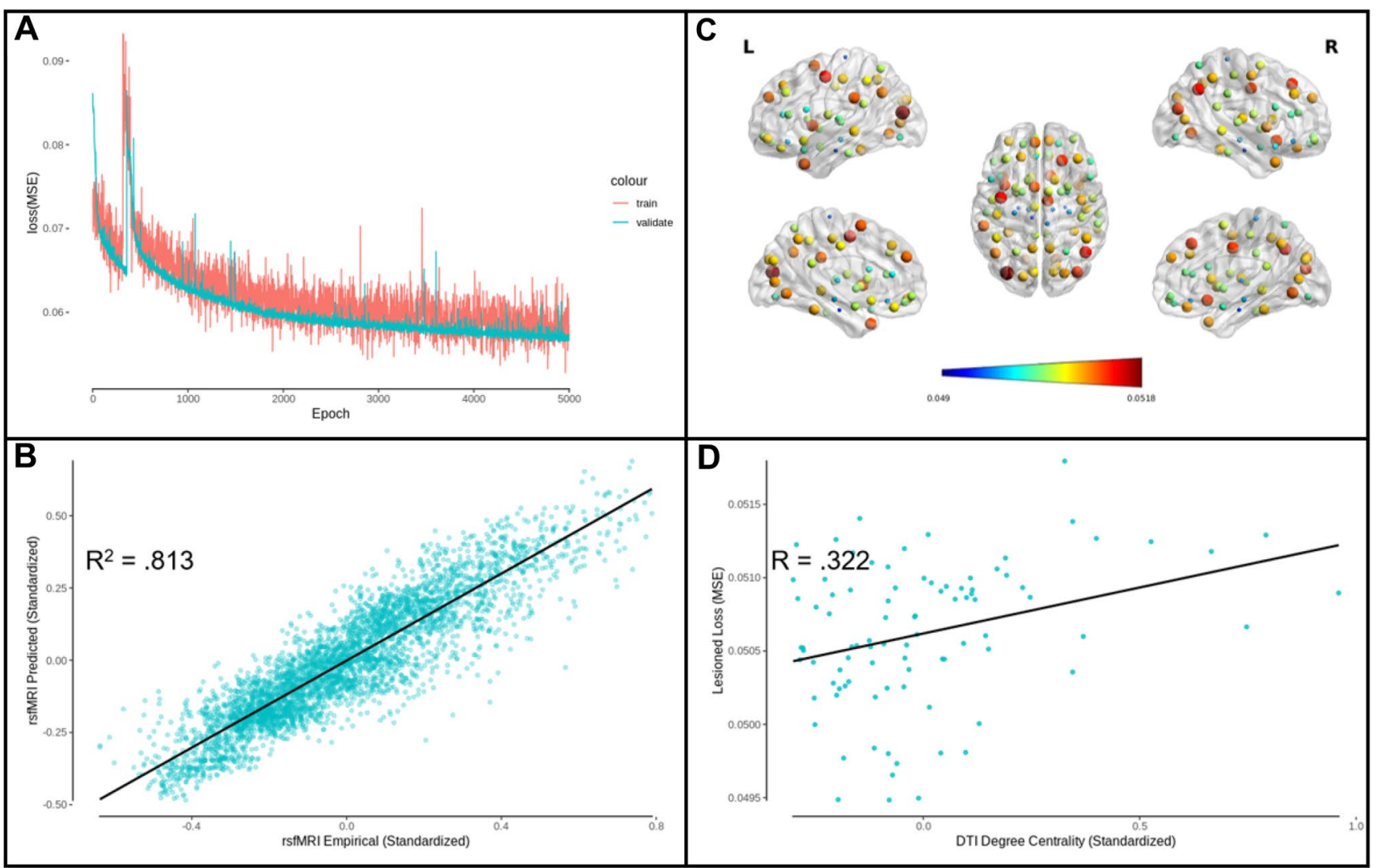

Fig. 3 AAL atlas edge prediction model performance. A Edge prediction model MSE loss for training and validation sets as a function of the number of training epochs. B Predicted rsfMRI functional connectivity as a function of empirical rsfMRI functional connectivity $\left(R^{2}=0.813\right)$. C Functional connectivity loss (MSE) related to lesioning structural connectivity to each atlas region, where dark blue indi-

Using the predicted functional connectivity, measures of centrality for degree, eigenvector, and PageRank were calculated by unstandardizing first before following the same thresholding and centrality calculation as described in the Methods. The mean predicted values for degree centrality accounted for $88.2 \%$ (DK) and $80.8 \%$ (AAL) of the variance in the empirical data (see Figs. 4A, 5A), for eigenvector centrality accounted for $89.9 \%$ (DK) and $84.0 \%$ (AAL) of the variance in the empirical data (see Figs. 4B, 5B), and for PageRank centrality accounted for $88.9 \%$ (DK) and $80.0 \%$ (AAL) of the variance in the empirical data (see Figs. 4C, $5 \mathrm{C})$. The individual-level predicted values for degree centrality accounted for $81.0 \%$ (DK) and $73.1 \%$ (AAL) of the variance in the empirical data (see Supplementary Figs. 3 and 4), for eigenvector centrality accounted for $55.3 \%$ (DK) and $53.0 \%$ (AAL) of the variance in the empirical data (see Supplementary Figs. 5 and 6), and for PageRank centrality accounted for $55.0 \%$ (DK) and $50.8 \%$ (AAL) of the variance in the empirical data (see Supplementary Figs. 7 and 8). These results demonstrate that the model has accounted for cates a lesser effect on the model performance and dark red indicates a greater effect. Larger sphere size also indicates a greater effect of lesion on model performance. Figure produced using BrainNet Viewer (Xia et al. 2013). D Lesioned functional connectivity loss as a function of structural connectivity degree centrality $(R(88)=0.322$, $p=.002)$

a large amount of variance in connectivity as well as centrality, but in order to determine if even better performance could be produced, the model designed to directly predict centrality was utilized.

\section{Degree centrality}

The degree prediction model was trained for 3000 epochs, and there was no evidence for overfitting of the model to the training set, as the validation set decreased its loss along with the training set (Figs. 6A, 7A). Figures 6B, 7B depict the empirical versus the predicted mean values of functional degree centrality, accounting for $99.3 \%$ (DK) and $99.0 \%$ (AAL) of the variance in the 201 subject test group. The individual-level functional degree centrality was predicted accounting for $63.7 \%$ (DK) and $64.7 \%$ (AAL) of the variance (Supplementary Figs. 9 and 10). This alternative model performed better than the previous model predicting the mean centrality, which accounted for $88.2 \%(\mathrm{DK})$ and $80.8 \%(\mathrm{AAL})$ of the variance, but 


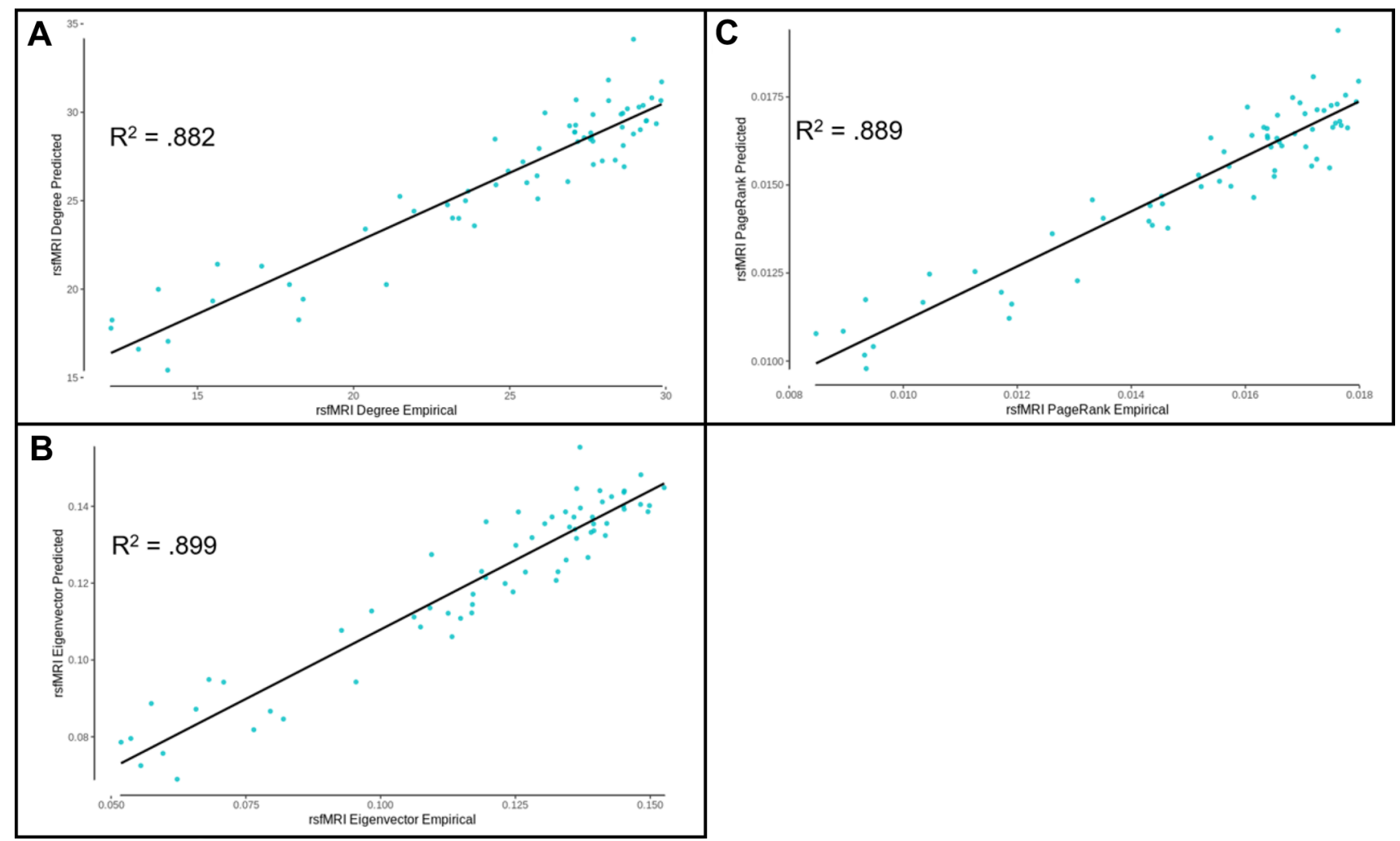

Fig. 4 DK atlas edge prediction model derived centrality measure performance. A Degree centrality measures calculated from the predicted functional connectivity values as a function of empirical degree centrality $\left(R^{2}=0.882\right)$. B Eigenvector centrality measures cal-

culated from the predicted functional connectivity values as a function of empirical eigenvector centrality $\left(R^{2}=.899\right)$. C PageRank centrality measures calculated from the predicted functional connectivity values as a function of empirical PageRank centrality $\left(R^{2}=0.889\right)$

performed worse than the previous model predicting the individual-level centrality, which accounted for $81.0 \%$ (DK) and $73.1 \%$ (AAL) of the variance.

\section{Eigenvector centrality}

The eigenvector prediction model was trained for 3000 epochs, and there was no evidence for overfitting of the model to the training set, as the validation set decreased its loss along with the training set (Figs. 6C, 7C). Figures $6 \mathrm{D}, 7 \mathrm{D}$ depict the empirical versus the predicted mean values of functional eigenvector centrality, accounting for $99.4 \%$ (DK) and $98.1 \%$ (AAL) of the variance in the 201 subject test group. The individual-level functional eigenvector centrality was predicted accounting for $62.3 \%$ (DK) and $61.2 \%$ (AAL) of the variance (Supplementary Figs. 11 and 12). This alternative model performed better than the previous model, which accounted for $89.9 \%$

(DK) and $84.0 \%$ (AAL) of the mean variance and $55.3 \%$ (DK) and $53.0 \%$ (AAL) of the individual-level variance.

\section{PageRank centrality}

The PageRank prediction model was trained for 3000 epochs, and there was no evidence for overfitting of the model to the training set, as the validation set decreased its loss along with the training set (Figs. 6E, 7E). Figures 6F, 7F depict the empirical versus the predicted mean values of functional PageRank centrality, accounting for $99.2 \%$ (DK) and $99.0 \%$ (AAL) of the variance in the 201 subject test group. The individual-level functional PageRank centrality was predicted accounting for $64.0 \%$ (DK) and 64.9\% (AAL) of the variance (Supplementary Figs. 13 and 14). This alternative model performed better than the previous model, which accounted for $88.9 \%$ (DK) and $80.0 \%$ (AAL) of the mean variance and $55.0 \%$ (DK) and $50.8 \%$ (AAL) of the individual-level variance. 


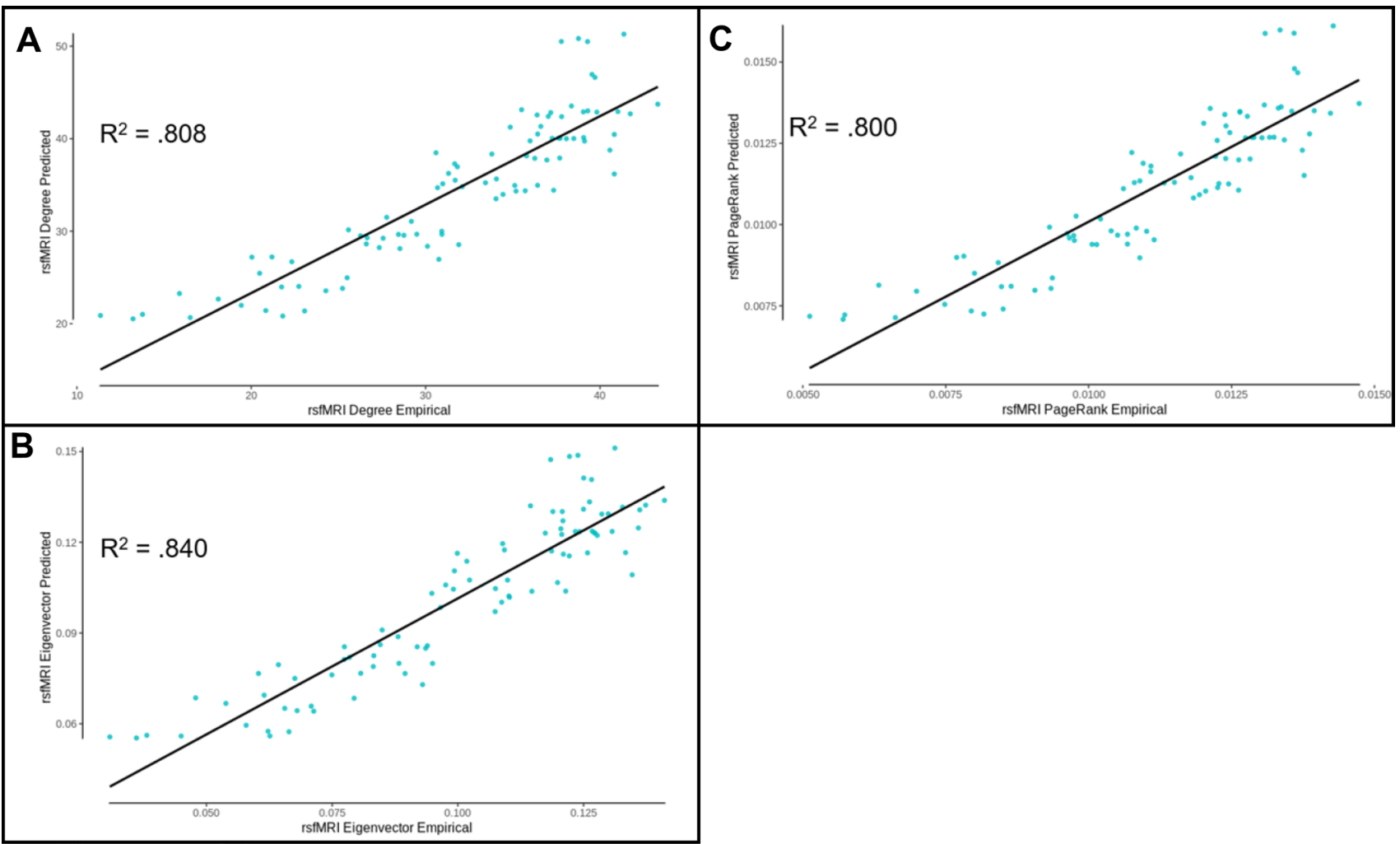

Fig. 5 AAL atlas edge prediction model derived centrality measure performance. A Degree centrality measures calculated from the predicted functional connectivity values as a function of empirical degree centrality $\left(R^{2}=0.808\right)$. B Eigenvector centrality measures calculated from the predicted functional connectivity values as

\section{Discussion}

Resting state fMRI has been used to calculate functional connectivity networks in brain research for many years, and graph theory measures such as centrality have been calculated for these functional networks, yet a clear relationship between the physical structural connectivity derived values and what is assumed to be the 'effective' functional connectivity has been elusive. The most successful previous effort to predict mean functional connectivity from mean structural connectivity at the whole-brain level was able to account for $81 \%$ of the variance in functional connectivity, and individual-level functional connectivity was predicted from individual-level structural connectivity accounting for $30 \%$ of the variance. The importance of continuing efforts to improve model prediction performance has been recently highlighted (Suárez et al. 2020). By using the Graph Nets (Battaglia et al. 2018) deep learning model architecture, which is well suited to modeling network datasets, mean functional connectivity was predicted from individual-level structural connectivity accounting for $89 \%$ of the variance (surpassing the previous attempt, without relying on mean structural a function of empirical eigenvector centrality $\left(R^{2}=0.840\right)$. C PageRank centrality measures calculated from the predicted functional connectivity values as a function of empirical PageRank centrality $\left(R^{2}=0.800\right)$

connectivity as input), and individual-level functional connectivity was predicted from individual-level structural connectivity accounting for $48 \%$ of the variance (far surpassing the previous attempt). In addition, mean functional centrality was predicted from individual-level structural connectivity and centrality data accounting for up to $99 \%$ of the variance, and up to $81 \%$ of individual-level functional centrality variance was accounted for from individual-level structural connectivity, demonstrating that these functional centrality measures can be robustly derived from the underlying structural connectivity and structural centrality measures. These results demonstrate that it is possible to account for nearly all of the mean-level variance in functional centrality with structural connectivity and centrality measures, suggesting that by calculating graph theory measures, information from the whole network is integrated, bridging the gap that is much more difficult to cross between structure and function at the edge level, and should encourage further research to explicitly define the nature of this relationship using graph theory and simulation modelling. Note that there is still variance left to be accounted for in predicting centrality at the individual level, which is not surprising considering that 


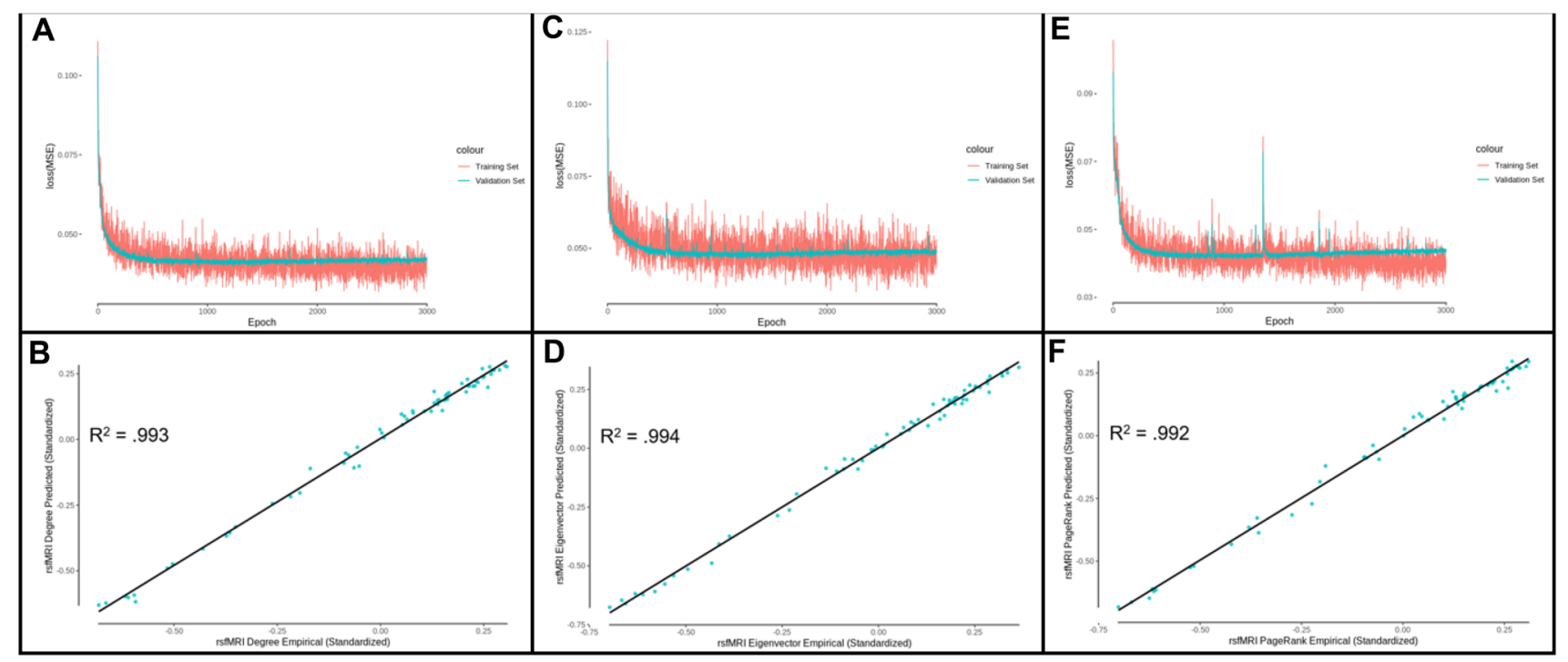

Fig. 6 DK atlas centrality prediction model performance. A Degree centrality model MSE loss for training and validation sets as a function of the number of training epochs. B Predicted rsfMRI functional connectivity degree centrality as a function of empirical rsfMRI functional connectivity degree centrality $\left(R^{2}=0.993\right)$. C Eigenvector centrality model MSE loss for training and validation sets as a function of the number of training epochs. D Predicted rsfMRI functional connectivity eigenvector centrality as a function of empirical rsfMRI functional connectivity eigenvector centrality $\left(R^{2}=0.994\right)$. E PageRank centrality model MSE loss for training and validation sets as a function of the number of training epochs. F Predicted rsfMRI functional connectivity PageRank centrality as a function of empirical rsfMRI functional connectivity PageRank centrality $\left(R^{2}=0.992\right)$

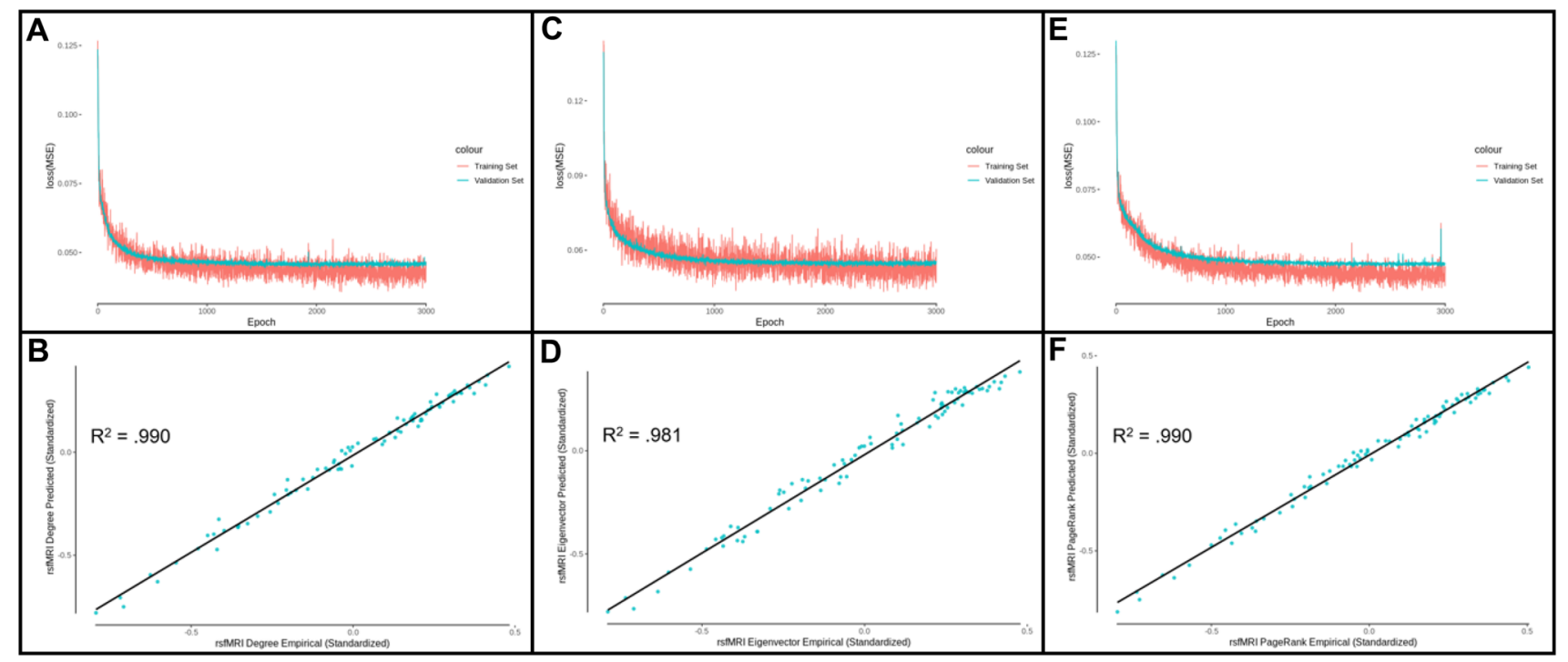

Fig. 7 AAL atlas centrality prediction model performance. A Degree centrality model MSE loss for training and validation sets as a function of the number of training epochs. B Predicted rsfMRI functional connectivity degree centrality as a function of empirical rsfMRI functional connectivity degree centrality $\left(R^{2}=0.990\right)$. C Eigenvector centrality model MSE loss for training and validation sets as a function of the number of training epochs. D Predicted rsfMRI functional

local structural connectivity at the microscale very likely contributes to functional centrality in a way that cannot be captured using macroscale methods. These results set an connectivity eigenvector centrality as a function of empirical rsfMRI functional connectivity eigenvector centrality $\left(R^{2}=0.981\right)$. E PageRank centrality model MSE loss for training and validation sets as a function of the number of training epochs. F Predicted rsfMRI functional connectivity PageRank centrality as a function of empirical rsfMRI functional connectivity PageRank centrality $\left(R^{2}=0.990\right)$

important benchmark in what should be a continuing effort in the network neuroscience community to determine the extent to which functional connectivity and graph theory 
measures such as centrality can be derived from structural connectivity, and therefore to what extent we can infer functional connectivity from structural connectivity.

The regions of the brain that are particularly important for the performance of the edge prediction model were highlighted by iteratively lesioning each region. In order to test whether the centrality may affect how important a region is to model performance, degree centrality was compared to lesion loss. Indeed, higher centrality was associated with greater importance for model performance, indicating that part of what makes a region more important to the model is the extent to which the region is centrally important to the network, connected to many other regions. As with all deep learning approaches, the limitations of this model include the nonlinear, underlying function that acts on the structural connectivity to predict the functional connectivity. Although we have addressed this limitation in part by showing a brain map of the regional importance to model performance via iterative lesioning, future research is needed using explicit graph theory measures and simulation models to define the nature of the structure-function relationship.

\section{Future directions}

The graph neural network deep learning model architecture is one that is designed with networks in mind, and maintains the structure of the brain connectivity data, whereas other commonly used deep learning approaches do not. We have demonstrated that this is an effective deep learning model for use with structural and functional connectivity data, whether the edge-, node-, or participant-level measures are of interest. Future research predicting patient, demographic, or behavioural data should also make use of this method in order to improve on past deep learning attempts using the "global" participant-level output available for training in the graph neural network model. Prediction of task activation from structural connectivity has also been explored in recent research using linear regression modelling approaches (e.g., Ekstrand et al. 2020; Osher et al. 2016; Wu et al. 2020). The use of a graph neural network model to predict fMRI task activation from structural connectivity represents an important advancement that could lead to improvements in task activation prediction.

As this model is further developed to account for more variance in individual-level functional connectivity, this approach may also lead to predictive functional mapping for patients who are unable to follow fMRI instructions. Alternatively, there may be an upper limit on the amount of information about functional connectivity that is contained in structural connectivity measures. This upper limit may exist due to a number of factors. Neuromodulation selectively inhibits and excites neurons throughout the brain network, leading to dynamic patterns of functional connectivity, so although the underlying structure does not change during MRI scanning, the functional connectivity is in constant flux (Bell and Shine 2016; Shine 2019). The low spatial resolution of the atlas used also means that the precise structural and functional connectivity patterns between neurons cannot be measured. In addition, functional boundaries vary across individuals and between sessions, as does the exact location of structural connections (Gordon et al. 2017; Laumann et al. 2015; Mueller et al. 2013; Salehi et al. 2020; Suárez et al. 2020; Wang et al. 2015), so the use of atlases is a source of error for this reason as well. The temporal dynamics also differ from one region to the next, affecting the calculation of functional connectivity (Gollo et al. 2015; Keitel and Gross 2016; Murray et al. 2014; Shafiei et al. 2019; Suárez et al. 2020). Depending on how unimodal or transmodal a region is, there may be a higher (unimodal) or lower (transmodal) degree of coupling between structure and function (Margulies et al. 2016; Preti and Van De Ville 2019; Suárez et al. 2020; Vázquez-Rodríguez et al. 2019). Based on our deep learning model results, there is clearly a large amount of variance in functional connectivity that can be accounted for by structural connectivity. Although the relationship discovered by deep learning is difficult to interpret directly, further graph theory and simulation modeling research can define this relationship explicitly, accounting for increased variance until maximal levels of variance are accounted for.

\section{Conclusion}

Our graph neural network deep learning model provides a new benchmark for prediction of rsfMRI functional connectivity ( $89 \%$ of mean variance; $56 \%$ of individual-level variance) and functional centrality (99\% of mean variance; $81 \%$ of individual-level variance) from DTI structural connectivity, which far exceeds the individual-level performance of the non graph neural network models previously reported by others ( $81 \%$ of mean variance; $30 \%$ of individual-level variance). This research has not only brought us closer to finding the upper limit on prediction of connectivity and centrality of function from structural connectivity, it has opened new doors for understanding the structure-function network relationships in the human brain.

Supplementary Information The online version contains supplementary material available at https://doi.org/10.1007/s00429-021-02403-8.

Acknowledgements This research was supported by the Natural Sciences and Engineering Research Council of Canada through Alexander Graham Bell Canada Graduate Scholarships to Josh Neudorf and Shaylyn Kress, and Discovery Grant 18968-2013-22 to the senior author Ron Borowsky. The authors affirm that there are no conflicts of interest to disclose. Data were provided [in part] by the Human Connectome 
Project, WU-Minn Consortium (Principal Investigators: David Van Essen and Kamil Ugurbil; 1U54MH091657) funded by the 16 NIH Institutes and Centers that support the NIH Blueprint for Neuroscience Research; and by the McDonnell Center for Systems Neuroscience at Washington University.

Data availability All data is made publicly available by the Human Connectome Project (Van Essen et al. 2013).

Code availability The Graph Nets (Battaglia et al. 2018) python library is accessible at https://github.com/albertotono/graph_nets. The code used to apply this library is available upon reasonable request.

\section{Declarations}

Conflict of interest The authors report no conflicts of interest.

Ethical approval, consent to participate and consent for publication Please see Van Essen et al. (2013) for a description of ethics and consent.

Open Access This article is licensed under a Creative Commons Attribution 4.0 International License, which permits use, sharing, adaptation, distribution and reproduction in any medium or format, as long as you give appropriate credit to the original author(s) and the source, provide a link to the Creative Commons licence, and indicate if changes were made. The images or other third party material in this article are included in the article's Creative Commons licence, unless indicated otherwise in a credit line to the material. If material is not included in the article's Creative Commons licence and your intended use is not permitted by statutory regulation or exceeds the permitted use, you will need to obtain permission directly from the copyright holder. To view a copy of this licence, visit http://creativecommons.org/licenses/by/4.0/.

\section{References}

Abadi M, Agarwal A, Barham P, Brevdo E Chen, Z, Citro C, Corrado GS, Davis A, Dean J, Devin M, Ghemawat S, Goodfellow I, Harp A, Irving G, Isard M, Jia Y, Jozefowicz R, Kaiser L, Kudlur M, Levenberg J, Mane D, Monga R, Moore S, Murray D, Olah C, Schuster M, Shlens J, Steiner B, Sutskever I, Talwar K, Tucker P, Vanhoucke V, Vasudevan V, Viegas F, Vinyals O, Warden P, Wattenberg M., Wicke M, Yu Y, Zheng X (2015) TensorFlow: large-scale machine learning on heterogeneous distributed systems. https://www.tensorflow.org/

Arslan S, Ktena SI, Glocker B, Rueckert D (2018) Graph saliency maps through spectral convolutional networks: application to sex classification with brain connectivity. In: Stoyanov D, Taylor Z, Ferrante E, Dalca AV, Martel A, Maier-Hein L, Parisot S, Sotiras A, Papiez B, Sabuncu MR, Shen L (eds) Graphs in biomedical image analysis and integrating medical imaging and non-imaging modalities lecture notes in computer science. Springer International Publishing, Cham, pp 3-13

Arya D, Olij R, Gupta DK, Gazzar AE, Wingen G, Worring M, Thomas RM (2020) Fusing structural and functional mris using graph convolutional networks for autism classification medical imaging with deep learning presented at the medical imaging with deep learning. PMLR, pp 44-61

Battaglia PW, Hamrick JB, Bapst V, Sanchez-Gonzalez A, Zambaldi V, Malinowski M, Tacchetti A, Raposo D, Santoro A, Faulkner
R, Gulcehre C, Song F, Ballard A, Gilmer J, Dahl G, Vaswani A, Allen K, Nash C, Langston V, Dyer C, Heess N, Wierstra D, Kohli P, Botvinick M, Vinyals O, Li Y, Pascanu R (2018) Relational inductive biases, deep learning, and graph networks. ArXiv180601261 Cs Stat

Bell PT, Shine JM (2016) Subcortical contributions to large-scale network communication. Neurosci Biobehav Rev 71:313-322. https://doi.org/10.1016/j.neubiorev.2016.08.036

Bi X, Liu Z, He Y, Zhao X, Sun Y, Liu H (2020) GNEA: a graph neural network with ELM aggregator for brain network classification. Complexity 2020:8813738. https://doi.org/10.1155/2020/8813738

Birn RM, Smith MA, Jones TB, Bandettini PA (2008) The respiration response function: the temporal dynamics of fMRI signal fluctuations related to changes in respiration. Neuroimage 40:644-654. https://doi.org/10.1016/j.neuroimage.2007.11.059

Chang C, Cunningham JP, Glover GH (2009) Influence of heart rate on the BOLD signal: the cardiac response function. Neuroimage 44:857-869. https://doi.org/10.1016/j.neuroimage.2008.09.029

Chen C, Wang H-L, Wu S-H, Huang H, Zou J-L, Chen J, Jiang T-Z, Zhou Y, Wang G-H (2015) Abnormal degree centrality of bilateral putamen and left superior frontal gyrus in schizophrenia with auditory hallucinations: a resting-state functional magnetic resonance imaging study. Chin Med J (engl) 128:3178-3184. https:// doi.org/10.4103/0366-6999.170269

Deng W, Zhang B, Zou W, Zhang X, Cheng X, Guan L, Lin Y, Lao G, Ye B, Li X, Yang C, Ning Y, Cao L (2019) Abnormal degree centrality associated with cognitive dysfunctions in early bipolar disorder. Front Psychiatry. https://doi.org/10.3389/fpsyt.2019.00140

Desikan RS, Ségonne F, Fischl B, Quinn BT, Dickerson BC, Blacker D, Buckner RL, Dale AM, Maguire RP, Hyman BT, Albert MS, Killiany RJ (2006) An automated labeling system for subdividing the human cerebral cortex on MRI scans into gyral based regions of interest. Neuroimage 31:968-980. https://doi.org/10.1016/j. neuroimage.2006.01.021

Destrieux C, Fischl B, Dale A, Halgren E (2010) Automatic parcellation of human cortical gyri and sulci using standard anatomical nomenclature. Neuroimage 53:1-15. https://doi.org/10.1016/j. neuroimage.2010.06.010

Ekstrand C, Neudorf J, Kress S, Borowsky R (2020) Structural connectivity predicts functional activation during lexical and sublexical reading. Neuroimage 218:117008. https://doi.org/10.1016/j.neuro image.2020.117008

Estrada E, Hatano N (2008) Communicability in complex networks. Phys Rev E 77:036111. https://doi.org/10.1103/PhysRevE.77. 036111

Falahpour M, Refai H, Bodurka J (2013) Subject specific BOLD fMRI respiratory and cardiac response functions obtained from global signal. Neuroimage 72:252-264. https://doi.org/10.1016/j.neuro image.2013.01.050

Fornito A, Zalesky A, Breakspear M (2013) Graph analysis of the human connectome: promise, progress, and pitfalls. NeuroImage 80:426-444. https://doi.org/10.1016/j.neuroimage.2013.04.087

Glasser MF, Sotiropoulos SN, Wilson JA, Coalson TS, Fischl B, Andersson JL, Xu J, Jbabdi S, Webster M, Polimeni JR, Van Essen DC, Jenkinson M (2013) The minimal preprocessing pipelines for the Human Connectome Project. NeuroImage 80:105-124. https:// doi.org/10.1016/j.neuroimage.2013.04.127

Golestani AM, Chang C, Kwinta JB, Khatamian YB, Jean Chen J (2015) Mapping the end-tidal CO2 response function in the resting-state BOLD fMRI signal: spatial specificity, test-retest reliability and effect of fMRI sampling rate. Neuroimage 104:266-277. https://doi.org/10.1016/j.neuroimage.2014.10.031

Gollo LL, Zalesky A, Hutchison RM, van den Heuvel M, Breakspear M (2015) Dwelling quietly in the rich club: brain network determinants of slow cortical fluctuations. Philos Trans R Soc B Biol Sci 370:20140165. https://doi.org/10.1098/rstb.2014.0165 
Goñi J, van den Heuvel MP, Avena-Koenigsberger A, de Mendizabal NV, Betzel RF, Griffa A, Hagmann P, Corominas-Murtra B, Thiran J-P, Sporns O (2014) Resting-brain functional connectivity predicted by analytic measures of network communication. Proc Natl Acad Sci 111:833-838. https://doi.org/10.1073/pnas.13155 29111

Gordon EM, Laumann TO, Gilmore AW, Newbold DJ, Greene DJ, Berg JJ, Ortega M, Hoyt-Drazen C, Gratton C, Sun H, Hampton JM, Coalson RS, Nguyen AL, McDermott KB, Shimony JS, Snyder AZ, Schlaggar BL, Petersen SE, Nelson SM, Dosenbach NUF (2017) Precision functional mapping of individual human brains. Neuron 95:791-807.e7. https://doi.org/10.1016/j.neuron. 2017.07.011

Hagberg AA, Schult DA, Swart PJ (2008) Exploring network structure, dynamics, and function using network. https://conference.scipy. org/proceedings/SciPy2008/paper_2/full_text.pdf

Hagmann P, Cammoun L, Gigandet X, Meuli R, Honey CJ, Wedeen VJ, Sporns O (2008) Mapping the structural core of human cerebral cortex. PLOS Biol 6:e159. https://doi.org/10.1371/journal. pbio.0060159

Hallquist MN, Hwang K, Luna B (2013) The nuisance of nuisance regression: spectral misspecification in a common approach to resting-state fMRI preprocessing reintroduces noise and obscures functional connectivity. Neuroimage 82:208-225. https://doi.org/ 10.1016/j.neuroimage.2013.05.116

Honey CJ, Sporns O, Cammoun L, Gigandet X, Thiran JP, Meuli R, Hagmann P (2009) Predicting human resting-state functional connectivity from structural connectivity. Proc Natl Acad Sci 106:2035-2040. https://doi.org/10.1073/pnas.0811168106

Kassinopoulos M, Mitsis GD (2019) Identification of physiological response functions to correct for fluctuations in resting-state fMRI related to heart rate and respiration. Neuroimage 202:116150. https://doi.org/10.1016/j.neuroimage.2019.116150

Keitel A, Gross J (2016) Individual human brain areas can be identified from their characteristic spectral activation fingerprints. PLOS Biol 14:e1002498. https://doi.org/10.1371/journal.pbio.1002498

Kingma DP, Ba J (2017) Adam: a method for stochastic optimization. ArXiv14126980 Cs

Klein A, Tourville J (2012) 101 Labeled Brain Images and a Consistent Human Cortical Labeling Protocol. Front Neurosci. https://doi. org/10.3389/fnins.2012.00171

Ktena SI, Parisot S, Ferrante E, Rajchl M, Lee M, Glocker B, Rueckert D (2017) Distance metric learning using graph convolutional networks: application to functional brain networks. In: Descoteaux M, Maier-Hein L, Franz A, Jannin P, Collins DL, Duchesne S (eds) Medical image computing and computer assisted intervention-MICCAI 2017, lecture notes in computer science. Springer International Publishing, Cham, pp 469-477

Laumann TO, Gordon EM, Adeyemo B, Snyder AZ, Joo SJ, Chen M-Y, Gilmore AW, McDermott KB, Nelson SM, Dosenbach NUF, Schlaggar BL, Mumford JA, Poldrack RA, Petersen SE (2015) Functional system and areal organization of a highly sampled individual human brain. Neuron 87:657-670. https://doi.org/10. 1016/j.neuron.2015.06.037

Lin Q, Zhu F-Y, Shu Y-Q, Zhu P-W, Ye L, Shi W-Q, Min Y-L, Li B, Yuan Q, Shao Y (2021) Altered brain network centrality in middle-aged patients with retinitis pigmentosa: a resting-state functional magnetic resonance imaging study. Brain Behav 11:e01983. https://doi.org/10.1002/brb3.1983

Long X, Little G, Treit S, Beaulieu C, Gong G, Lebel C (2020) Altered brain white matter connectome in children and adolescents with prenatal alcohol exposure. Brain Struct Funct 225:1123-1133. https://doi.org/10.1007/s00429-020-02064-z

Margulies DS, Ghosh SS, Goulas A, Falkiewicz M, Huntenburg JM, Langs G, Bezgin G, Eickhoff SB, Castellanos FX, Petrides M, Jefferies E, Smallwood J (2016) Situating the default-mode network along a principal gradient of macroscale cortical organization. Proc Natl Acad Sci 113:12574-12579. https://doi.org/10.1073/ pnas. 1608282113

Messé A (2020) Parcellation influence on the connectivity-based structure-function relationship in the human brain. Hum Brain Mapp 41:1167-1180. https://doi.org/10.1002/hbm.24866

Mueller S, Wang D, Fox MD, Yeo BTT, Sepulcre J, Sabuncu MR, Shafee R, Lu J, Liu H (2013) Individual variability in functional connectivity architecture of the human brain. Neuron 77:586-595. https://doi.org/10.1016/j.neuron.2012.12.028

Murray JD, Bernacchia A, Freedman DJ, Romo R, Wallis JD, Cai X, Padoa-Schioppa C, Pasternak T, Seo H, Lee D, Wang X-J (2014) A hierarchy of intrinsic timescales across primate cortex. Nat Neurosci 17:1661-1663. https://doi.org/10.1038/nn.3862

Neudorf J, Ekstrand C, Kress S, Borowsky R (2020) Brain structural connectivity predicts brain functional complexity: diffusion tensor imaging derived centrality accounts for variance in fractal properties of functional magnetic resonance imaging signal. Neuroscience 438:1-8. https://doi.org/10.1016/j.neuroscience.2020.04.048

Osher DE, Saxe RR, Koldewyn K, Gabrieli JDE, Kanwisher N, Saygin ZM (2016) Structural connectivity fingerprints predict cortical selectivity for multiple visual categories across cortex. Cereb Cortex 26:1668-1683. https://doi.org/10.1093/cercor/bhu303

Page L, Brin S, Motwani R, Winograd T (1999) The PageRank Citation Ranking: Bringing Order to the Web. [WWW Document]. URL http://ilpubs.stanford.edu:8090/422/ (Accessed 9.23.19)

Parisot S, Ktena SI, Ferrante E, Lee M, Guerrero R, Glocker B, Rueckert D (2018) Disease prediction using graph convolutional networks: application to autism spectrum disorder and Alzheimer's disease. Med Image Anal 48:117-130. https://doi.org/10.1016/j. media.2018.06.001

Preti MG, Van De Ville D (2019) Decoupling of brain function from structure reveals regional behavioral specialization in humans. Nat Commun 10:4747. https://doi.org/10.1038/s41467-019-12765-7

Raizman R, Tavor I, Biegon A, Harnof S, Hoffmann C, Tsarfaty G, Fruchter E, Tatsa-Laur L, Weiser M, Livny A (2020) Traumatic brain injury severity in a network perspective: a diffusion mri based connectome study. Sci Rep 10:9121. https://doi.org/10. 1038/s41598-020-65948-4

Rosenthal G, Váša F, Griffa A, Hagmann P, Amico E, Goñi J, Avidan G, Sporns O (2018) Mapping higher-order relations between brain structure and function with embedded vector representations of connectomes. Nat Commun 9:1-12. https://doi.org/10.1038/ s41467-018-04614-w

Salas JA, Bayrak RG, Huo Y, Chang C (2021) Reconstruction of respiratory variation signals from fMRI data. Neuroimage 225:117459. https://doi.org/10.1016/j.neuroimage.2020.117459

Salehi M, Greene AS, Karbasi A, Shen X, Scheinost D, Constable RT (2020) There is no single functional atlas even for a single individual: Functional parcel definitions change with task. Neuroimage 208:116366. https://doi.org/10.1016/j.neuroimage.2019.116366

Salimi-Khorshidi G, Douaud G, Beckmann CF, Glasser MF, Griffanti L, Smith SM (2014) Automatic denoising of functional mri data: combining independent component analysis and hierarchical fusion of classifiers. Neuroimage 90:449-468. https://doi.org/10. 1016/j.neuroimage.2013.11.046

Sarwar T, Tian Y, Yeo BTT, Ramamohanarao K, Zalesky A (2021) Structure-function coupling in the human connectome: a machine learning approach. Neuroimage 226:117609. https://doi.org/10. 1016/j.neuroimage.2020.117609

Schirner M, McIntosh AR, Jirsa V, Deco G, Ritter P (2018) Inferring multi-scale neural mechanisms with brain network modelling. ELife 7:e28927. https://doi.org/10.7554/eLife.28927

Shafiei G, Zeighami Y, Clark CA, Coull JT, Nagano-Saito A, Leyton M, Dagher A, Mišić B (2019) Dopamine signaling modulates the 
stability and integration of intrinsic brain networks. Cereb Cortex 29:397-409. https://doi.org/10.1093/cercor/bhy264

Shine JM (2019) Neuromodulatory influences on integration and segregation in the brain. Trends Cogn Sci 23:572-583. https://doi. org/10.1016/j.tics.2019.04.002

Suárez LE, Markello RD, Betzel RF, Misic B (2020) Linking structure and function in macroscale brain networks. Trends Cogn Sci 24:302-315. https://doi.org/10.1016/j.tics.2020.01.008

Turkiewicz J, Bhatt RR, Wang H, Vora P, Krause B, Sauk JS, Jacobs JP, Bernstein CN, Kornelsen J, Labus JS, Gupta A, Mayer EA (2021) Altered brain structural connectivity in patients with longstanding gut inflammation is correlated with psychological symptoms and disease duration. NeuroImage Clin. https://doi.org/10.1016/j. nicl.2021.102613

Tzourio-Mazoyer N, Landeau B, Papathanassiou D, Crivello F, Etard O, Delcroix N, Mazoyer B, Joliot M (2002) Automated anatomical labeling of activations in SPM using a macroscopic anatomical parcellation of the MNI MRI single-subject brain. Neuroimage 15:273-289. https://doi.org/10.1006/nimg.2001.0978

van den Heuvel MP, Hulshoff Pol HE (2010) Exploring the brain network: a review on resting-state fMRI functional connectivity. Eur Neuropsychopharmacol 20:519-534. https://doi.org/10.1016/j. euroneuro.2010.03.008

Van Essen DC, Smith SM, Barch DM, Behrens TEJ, Yacoub E, Ugurbil K (2013) The WU-minn human connectome project: an overview. NeuroImage 80:62-79. https://doi.org/10.1016/j.neuroimage. 2013.05.041

Vázquez-Rodríguez B, Suárez LE, Markello RD, Shafiei G, Paquola C, Hagmann P, van den Heuvel MP, Bernhardt BC, Spreng RN, Misic B (2019) Gradients of structure-function tethering across neocortex. Proc Natl Acad Sci USA 116:21219-21227. https:// doi.org/10.1073/pnas.1903403116

Wang D, Buckner RL, Fox MD, Holt DJ, Holmes AJ, Stoecklein S, Langs G, Pan R, Qian T, Li K, Baker JT, Stufflebeam SM, Wang K, Wang X, Hong B, Liu H (2015) Parcellating cortical functional networks in individuals. Nat Neurosci 18:1853-1860. https://doi. org/10.1038/nn.4164

Wang X, Liang X, Jiang Z, Nguchu BA, Zhou Y, Wang Y, Wang H, Li Y, Zhu Y, Wu F, Gao J-H, Qiu B (2020) Decoding and mapping task states of the human brain via deep learning. Hum Brain Mapp 41:1505-1519. https://doi.org/10.1002/hbm.24891

Wang L, Li K, Hu XP (2021) Graph convolutional network for fMRI analysis based on connectivity neighborhood. Netw Neurosci 5:83-95. https://doi.org/10.1162/netn_a_00171

Wink AM, Tijms BM, ten Kate M, Raspor E, de Munck JC, Altena E, Ecay-Torres M, Clerigue M, Estanga A, Garcia-Sebastian M, Izagirre A, Martinez-Lage Alvarez P, Villanua J, Barkhof F, SanzArigita E (2018) Functional brain network centrality is related to APOE genotype in cognitively normal elderly. Brain Behav. https://doi.org/10.1002/brb3.1080

Wu D, Fan L, Song M, Wang H, Chu C, Yu S, Jiang T (2020) Hierarchy of connectivity-function relationship of the human cortex revealed through predicting activity across functional domains. Cereb Cortex 30:4607-4616. https://doi.org/10.1093/cercor/bhaa063

Xia M, Wang J, He Y (2013) BrainNet viewer: a network visualization tool for human brain connectomics. PLoS ONE 8:e68910. https:// doi.org/10.1371/journal.pone.0068910

Xu Q-H, Li Q-Y, Yu K, Ge Q-M, Shi W-Q, Li B, Liang R-B, Lin Q, Zhang Y-Q, Shao Y (2020) Altered brain network centrality in patients with diabetic optic neuropathy: a resting-state FMRI study. Endocr Pract 26:1399-1405. https://doi.org/10.4158/ EP-2020-0045

Yeh F, Wedeen VJ, Tseng WI (2010) Generalized $q$-sampling imaging. IEEE Trans Med Imaging 29:1626-1635. https://doi.org/10.1109/ TMI.2010.2045126

Yeh F-C, Verstynen TD, Wang Y, Fernández-Miranda JC, Tseng W-YI (2013) Deterministic diffusion fiber tracking improved by quantitative anisotropy. PLoS ONE. https://doi.org/10.1371/journal. pone.0080713

Yu Z, Tao L, Qian Z, Wu J, Liu H, Yu Y, Song J, Wang S, Sun J (2016) Altered brain anatomical networks and disturbed connection density in brain tumor patients revealed by diffusion tensor tractography. Int J Comput Assist Radiol Surg 11:2007-2019. https://doi. org/10.1007/s11548-015-1330-y

Zhang W, Wang Y (2020) Deep Multimodal Brain Network Learning for Joint Analysis of Structural Morphometry and Functional Connectivity. In: 2020 IEEE 17th International Symposium on Biomedical Imaging (ISBI). Presented at the 2020 IEEE 17th International Symposium on Biomedical Imaging (ISBI), pp. 1-5. https://doi.org/10.1109/ISBI45749.2020.9098624

Zhou Q, Womer FY, Kong L, Wu F, Jiang X, Zhou Y, Wang D, Bai C, Chang M, Fan G, Xu K, He Y, Tang Y, Wang F (2017) Traitrelated cortical-subcortical dissociation in bipolar disorder: analysis of network degree centrality. J Clin Psychiatry 78:584-591. https://doi.org/10.4088/JCP.15m10091

Zuo X-N, Ehmke R, Mennes M, Imperati D, Castellanos FX, Sporns O, Milham MP (2012) Network centrality in the human functional connectome. Cereb Cortex 22:1862-1875. https://doi.org/ 10.1093/cercor/bhr269

Publisher's Note Springer Nature remains neutral with regard to jurisdictional claims in published maps and institutional affiliations. 